\title{
Thermoelectric Energy Conversion of a Drinking Bird by Disk-Magnet Electromagnetic Induction
}

\author{
Hiroshi Uechi', Schun T. Uechi² \\ ${ }^{1}$ Osaka Gakuin University, Suita, Osaka, Japan \\ ${ }^{2}$ KPMG Ignition Tokyo, Data Tech., Tokyo, Japan \\ Email: uechi@ogu.ac.jp,quantum2006hara@gmail.com
}

How to cite this paper: Uechi, H. and Uechi, S.T. (2020) Thermoelectric Energy Conversion of a Drinking Bird by DiskMagnet Electromagnetic Induction. World Journal of Engineering and Technology, 8, 204-216.

https://doi.org/10.4236/wjet.2020.82017

Received: April 6, 2020

Accepted: May 4, 2020

Published: May 7, 2020

Copyright () 2020 by author(s) and Scientific Research Publishing Inc. This work is licensed under the Creative Commons Attribution International License (CC BY 4.0).

http://creativecommons.org/licenses/by/4.0/

\begin{abstract}
We discuss a thermoelectric energy generation (TEG) technique by employing a thermomechanical model of a drinking bird (DB). The motion of a drinking bird is produced by the entropy-flow explained by the second law of thermodynamics, which is one of the fundamental laws of heat engines. We propose a disk-magnet electromagnetic induction (DM-EMI) employed to the motion of a drinking bird. The generalization of DM-EMI to heat engines for mechanoelectric energy conversions and properties of extracted electric powers are specifically discussed. The electric power of DM-EMI has a limited power generation characteristic to a mechanical rotation produced by heat engines, but it will be very useful for practical applications to wind turbines, coal-fired and nuclear power plant for mechanoelectric energy conversions. The DM-EMI will contribute to environmental problems to maintain clean and susceptible energy as one of energy harvesting technologies.
\end{abstract}

\section{Keywords}

Mechanoelectric Energy Generation (MEG), Thermomechanical Model of a Drinking Bird (DB), Disk-Magnet Electromagnetic Induction (DM-EMI)

\section{Introduction}

A mathematical expression of motion for a thermomechanical drinking bird (DB) was discussed and numerical solutions were explicitly derived and shown [1]. A drinking bird is a model of irreversible thermodynamics and manifests basic principles of heat engines, which is useful for mechanoelectric energy conversions. The model reproduced a DB's periodic motion reasonably well with the assumption of a constant speed, $v_{0}$, of volatile liquid in a DB's glass tube. The 
result is somewhat surprising, because DB's motion is a simple back-and-force oscillating motion and the speed, $v_{0}$, of volatile water in the glass tube should not be constant. The result indicates that the mean-value assumption to the constant speed of upward moving liquid is reasonable for solving a DB's equation of motion as the first step.

Drinking birds and Stirling engines are excellent examples of heat engines or thermomechanical phenomena and should be scrutinized in terms of the theory of nonequilibrium thermodynamics or irreversible process of thermodynamics [2] [3]. The sensitive heat-flow or entropy-flow of a DB will help understand irreversible thermodynamic phenomena and testability of theoretical concepts, which could be, for example, extended and interpreted as a role of mitochondria in a cell or photo electrochemical processes in semiconductors [4] [5].

The upward moving liquid is essential for a DB's periodic motion, and the immediate driving force of upward moving liquid is a pressure in the glass tube, originated from a heat-flow, a temperature difference between upper head-bulb and lower bulb, or an entropy-flow. The analysis of sensitive heat engines is essential for effective and optimal uses of energies in mechanical applications and fundamental understanding of thermodynamic phenomena. One can learn fundamental principles of thermal reactors, or even applications to nuclear reactors from dynamics of heat engines of drinking birds and Stirling engines as one of energy conversion techniques of energy harvesting technologies [6] [7].

As explained in the previous paper for testability and applicability [1], we will show a specific thermally-driven mechanoelectric energy conversion technique derived from the sensitive irreversible thermodynamic structure of a drinking bird. The fundamental difference between nonlinear differential equations of Newtoninan and thermomechanical drinking birds is briefly discussed in Section 2. The disk-magnet electromagnetic induction by a drinking bird and an extended disk-magnet electromagnetic energy conversion technique for heat engines, in general, are discussed in Section 3. Discussions and perspectives are in Section 4.

\section{The Nonlinear Differential Equation of a Thermomechanical Drinking Bird}

The equation of motion for a mechanical drinking bird could be first constructed by employing Newtonian mechanics, Lagrangian or Hamiltonian dynamics with the rotational kinetic energy and potential energy:

$$
\mathcal{L}=\frac{1}{2} I_{0} \dot{\theta}^{2}+\left\{m_{2} g a-m_{1} g\left(b-\frac{l}{2}\right)-m_{3} g b\right\} \cos \theta .
$$

where the DB's length $l=a+b$, the moment of inertia given by the sum, $I_{0}=I_{1}+I_{2}+I_{3}$ which is moment of inertia of head $I_{3}$, glass tube $I_{1}$, and lower bulb $I_{2}$, respectively. By employing the idea of the center of mass assumption, the moment of inertia of head and lower bulb is given by $I_{2}=m_{2} a^{2}$ and $I_{3}=m_{3} b^{2}$; $I_{1}$ is calculated from the axis of rotation (see Figure $1(b)$ ). One should note that the mass, $m_{2}$, is the sum of masses of liquid and lower glass bulb. 


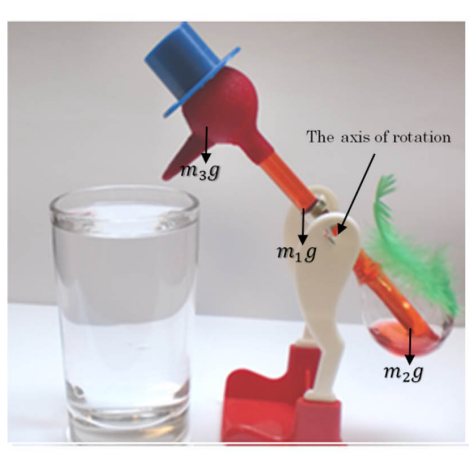

(a)

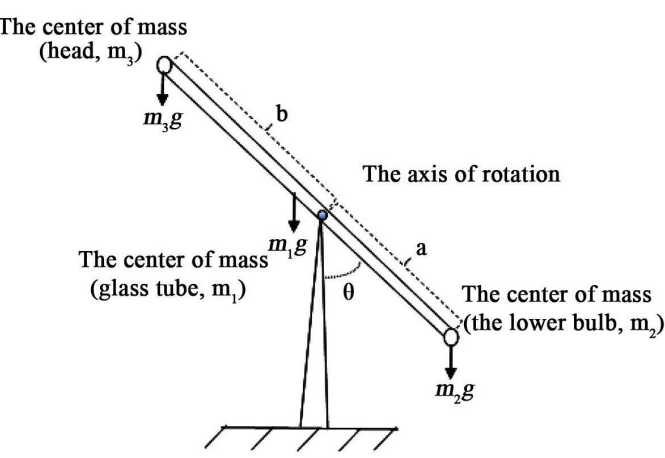

(b)

Figure 1. A mechanical modeling of a drinking bird. (a) A drinking bird (DB); (b) A mechanical model for the drinking bird of (a).

The mechanical equation of motion for DB is derived from the Euler-Lagrange equation as:

$$
I_{0} \ddot{\theta}+g l\left\{m_{2} \frac{a}{l}-m_{1}\left(\frac{b}{l}-\frac{1}{2}\right)-m_{3} \frac{b}{l}\right\} \sin \theta=0 .
$$

The velocity-dependent friction term, $c \dot{\theta}$ ( $c$ is a free parameter), is externally included in the equation of motion for accounting an oscillation damping, and by defining the effective mass, $m^{*}$, for convenience as:

$$
m^{*}=m_{2} \frac{a}{l}-m_{1}\left(\frac{b}{l}-\frac{1}{2}\right)-m_{3} \frac{b}{l},
$$

the mechanical equation of motion for a DB is written as:

$$
\ddot{\theta}+c \dot{\theta}+\frac{g l m^{*}}{I_{0}} \sin \theta=0 .
$$

The motion of a drinking bird is a combination of the solutions converging to $\theta=0$ and $\theta=\pi$. However, the mechanical solutions derived from (2.4) are linearly independent and cannot produce the motion of a drinking bird. The continuous drinking motion from $\theta=0$ to $\theta=\pi$ does not exist in (2.4), because the solutions converging to $\theta=0$ and $\theta=\pi$ are respectively bifurcation solutions [8].

We proposed a thermomechanical model of a DB's periodic motion assuming a constant speed, $v_{0}$, of volatile liquid in a DB's glass tube. The equation of motion for the thermomechanical DB results in,

$$
\ddot{\theta}+c \dot{\theta}+\frac{g l m^{*}(t)}{I(t)} \sin \theta=0,
$$

and the thermomechanical solution of (2.5) produces a DB's periodic motion reasonably well. The nonlinear differential Equation (2.5) should be compared with the mechanical equation of motion of (2.4). The Equation (2.4) and Equation (2.5) are almost identical in the sense that the equation of motion for the thermomechanical DB is identical to the mechanical equation of motion replaced as $\left(m^{*}, I_{0}\right) \rightarrow\left(m^{*}(t), I(t)\right)$ (see the paper [1] for detailed discussions). 
The constant speed of liquid, $v_{0}$, in the glass tube generates time-dependent masses, $m^{*}(t)$, and inertial masses, $I(t)$, producing a periodic DB's solution, and the speed of liquid $v_{0}$ is essential for the entire motion driven by entropy flow of DB's mechanical system.

The solutions of nonlinear differential equation with time-dependent coefficients are fundamentally different from those of nonlinear differential equation with time-independent coefficients (2.4). This is one of the important properties shown by DB's equation of motion. The numerical solutions to a drinking bird motion (2.5) are shown in Figure 2. We will use the result for a mechanoelectric energy conversion of the drinking bird motion in the next section.

\section{The Disk-Magnet Electromagnetic Induction by a Drinking Bird}

The energy generation from a drinking bird is a sensitive mechanoelectric energy conversion process, and it could suggest useful ideas for applications to modern energy harvesting technologies. The motion of a drinking bird was recorded with the use of modern equipments by experimentalists, but was not solved theoretically. We solved a thermomechanical model of nonlinear motion of a drinking bird [1] and are ready to propose a direct mechanoelectric conversion of DB's motion for applications. In this section, we discuss and show an optimal energy conversion technique by employing thermomechanical solutions of a drinking bird.

A generation of electric energy from a drinking bird was only experimentally tested by attaching a magnet and a coil on the base of a drinking bird [9], however the energy conversion method had a couple of unavoidable problems:

1) A coil-magnet dynamo on the base of a drinking bird will act as magnetic force to brake and damp the swinging motion. Though a better improvement of dynamo on the base of a DB is possible, the extraction of energy would ultimately yield diminishing results.

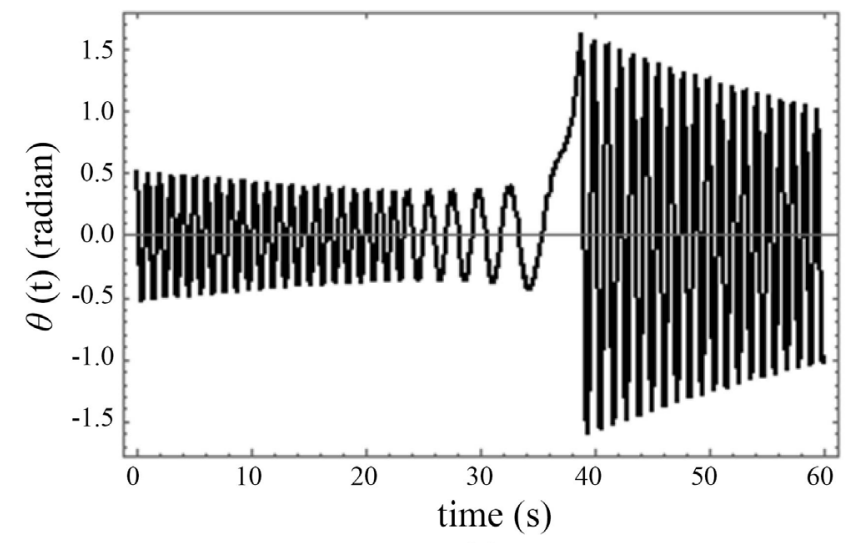

(a)

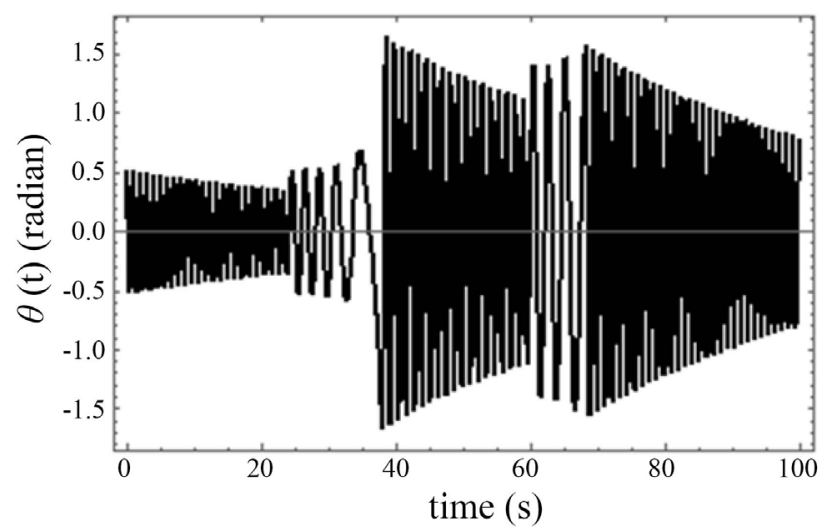

(b)

Figure 2. The drinking motion of a thermomechanical drinking bird calculated by Equation (2.5). (a) The bird's drinking motion at $t \simeq 38.8$ seconds and $\theta \simeq \pi / 2$ shows oscillations after drinking; (b) The first and second drinking motions of thermomechanical DB at $t_{1}=38.0$ and $t_{2}=71.5$. 
2) A coil-magnet dynamo on the base of a drinking bird will be soaked and affected by water dripping through the head to the lower bulb in drinking motion.

The difficulties (a magnetic braking of motion and water dripping) seem to be sufficient enough not to consider any practical applications in electric power generations.

However, we will show an optimal thermoelectric energy conversion technique by resolving above difficulties and extend the power conversion technique to heat engines from milli-watt to killo-watt electric power generations. The irreversible thermodynamic process expressed by a drinking bird and Stirling engines is useful for foundations and the progress of energy harvesting technologies.

The disk-magnet electromagnetic induction of symmetric settings of coils and magnets is shown in Figure 3, in order to solve the essential problem of coil-magnet dynamo on the base of a drinking bird. The magnets and coils are arranged horizontally on the right and left of the lower bulb of the drinking bird. The forces between a magnet and a coil exerted by right and left coils on DB's body work to cancel each other. Therefore, the braking or damping effects coming from electromagnetic induction forces can be eliminated. In other words, the produced magnetic forces which caused magnetic braking and damping of swinging motion cancel each other with the symmetric setting of magnets and coils.

For the disk-magnet electromagnetic induction when the magnet crosses near the cross-sectional area of a corresponding coil, a simplified assumption on the magnetic flux is employed. As shown in Figure 3(b), the magnetic flux is perpendicular to the magnetic disk, and the maximum magnetic flux penetrating a coil is given by magnetic flux density $B\left(\mathrm{~Wb} / \mathrm{cm}^{2}\right)$ multiplied by the cross-sectional

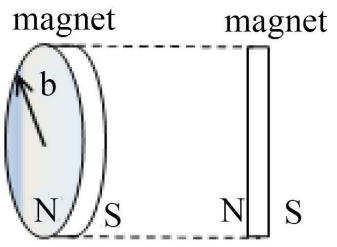

(a)

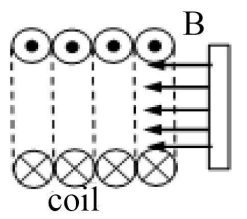

(b)

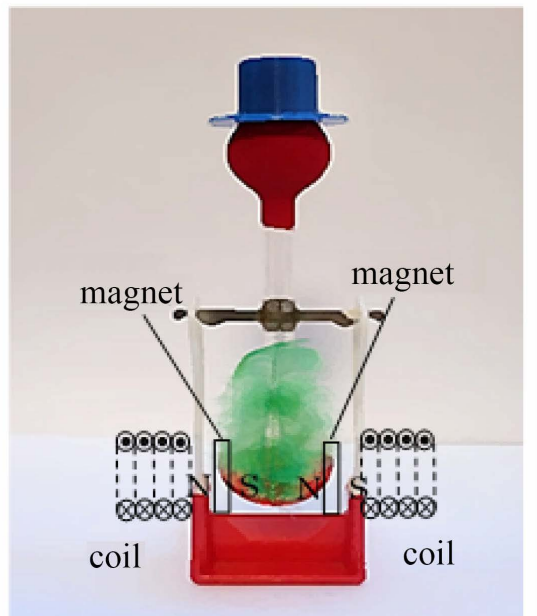

(c)

Figure 3. The energy conversion technique of a thermomechanical drinking bird from mechanical swinging motion to electric energy. (a) A disk magnet; (a) The magnetic flux $\mathrm{B}$; (c) The rear view with coils and magnets. 
area of coil, $S=\pi b^{2}\left(\mathrm{~cm}^{2}\right)$, as $B \cdot S(\mathrm{~Wb})$. The assumption is physically reasonable when the magnet is strong (e.g. neodymium magnet) and slides through near the cross-sectional area of the coil (see Figure $3(b)$ ).

The magnetic flux becomes time-dependent as $\phi(t)=B S(\theta(t))$ with the maximum flux, $\phi_{\max }=B \pi b^{2}$, and the pulsations of magnetic flux are extracted, generating those of electric currents. The detailed electromagnetic induction and magnetic flux $\phi(t)$, are calculated at a moment of a fixed-coil and magnet intersection as shown in Figure 4. The calculations of $\theta(t), \phi(\theta(t)), S(\theta(t))$ and pulses of electric currents are discussed in Appendix.

\subsection{Numerical Simulations of the Disk-Magnet Mechanoelectric Energy Conversion}

The pulses of electric currents are extracted by connecting a coil with an RLC circuit, and hence, the experimental values of the resistance, $R$, inductance, $L$, and capacity, $C$, winding numbers of coils, magnetic flux and the experimental values of a drinking bird are considered to be adjusted in order to qualitatively observe the mechanoelectric energy conversion. The validity of assumption and quantitative values of generated electric current and power should be checked by experiment.

A drinking bird exhibits a simple back and forth motion as shown in Figure 2, and the mechanoelectric energy-conversion simulations are performed with one of the right or left magnet-coil system, and so the total electric energy would be, roughly speaking, twice the results. The time range of motion is chosen for numerical demonstration purpose, which is taken for example, $20.0 \leq t \leq 22.0$ seconds, and the initial conditions are $\theta(0)=\pi / 3$ and $\theta^{\prime}(0)=0$. The solution of angle, $\theta(t)$, and accompanying pulse angles, $\Delta \theta(t)$, for mechanoelectric

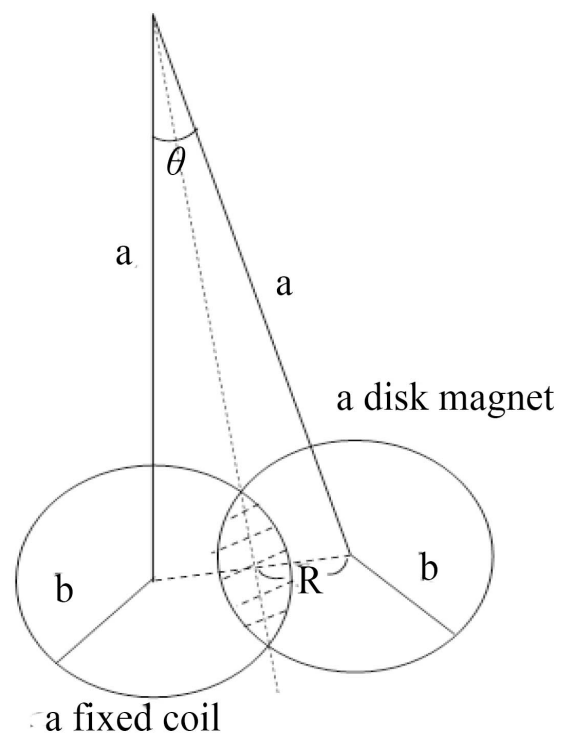

Figure 4. The image at a moment of an intersection for the coil cross-section and diskmagnet. The shaded area is $\phi(t)=B S(\theta(t)) \quad(\mathrm{Wb})$, and $R(t)=a \sin (\theta(t) / 2) \quad(\mathrm{cm})$. 
energy generations are shown in Figure 5(a) and Figure 5(b). One can check the corresponding pulses between $\theta(t)$ and $\Delta \theta(t)$ by comparing Figure 5(a) with Figure $5(\mathrm{~b})$. The pulse angles, $\Delta \theta(t)$, are parts of the solution, $\theta(t)$, around angle $\theta(t)=0$, which generates the pulse electric current, $\Delta I(t)$.

The pulse angle-velocities, $\mathrm{d} \theta / \mathrm{d} t(\mathrm{rad} / \mathrm{s})$, and the magnetic pulses, $B S(\theta(t))$ $(\mathrm{Wb})$, are shown in Figure 6. The smooth curve within each pulsation period shows the pulse angle-velocity in Figure 6(a), and the values of pulse angle-velocity alternate $\pm(3.5$ - 4.0) ( $\mathrm{rad} / \mathrm{s})$, associated to the DB's back and forth motion. One can check the values of pulse angle-velocity by changing initial conditions from $\left(\theta(0)=\pi / 6, \theta^{\prime}(0)=0\right)$ to $\left(\theta(0)=\pi / 3, \theta^{\prime}(0)=0\right)$. The pulse angle velocity with the starting value, $\theta(0)=\pi / 3$, becomes about 2 times larger than that the starting value, $\theta(0)=\pi / 6$.

However, one should note that it does not mean that the larger the angular speed, $\mathrm{d} \theta(t) / \mathrm{d} t$, results in the larger pulse electric current and power in DM-EMI.

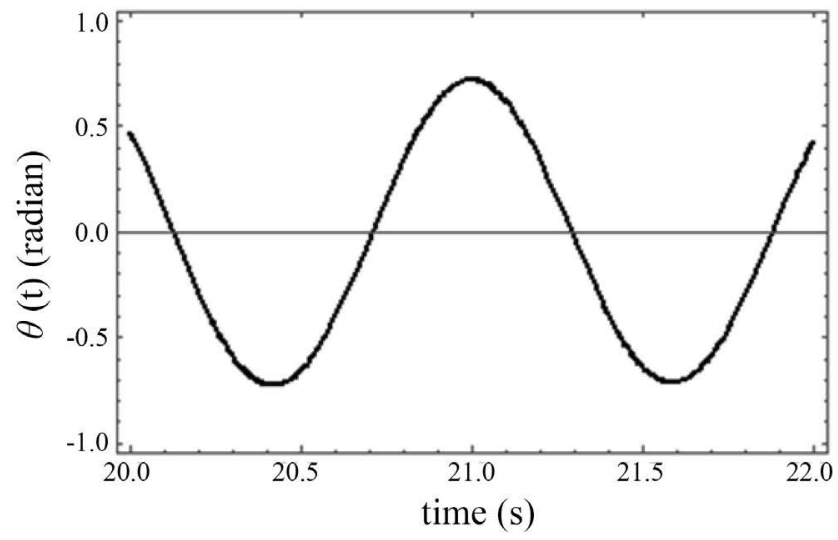

(a)

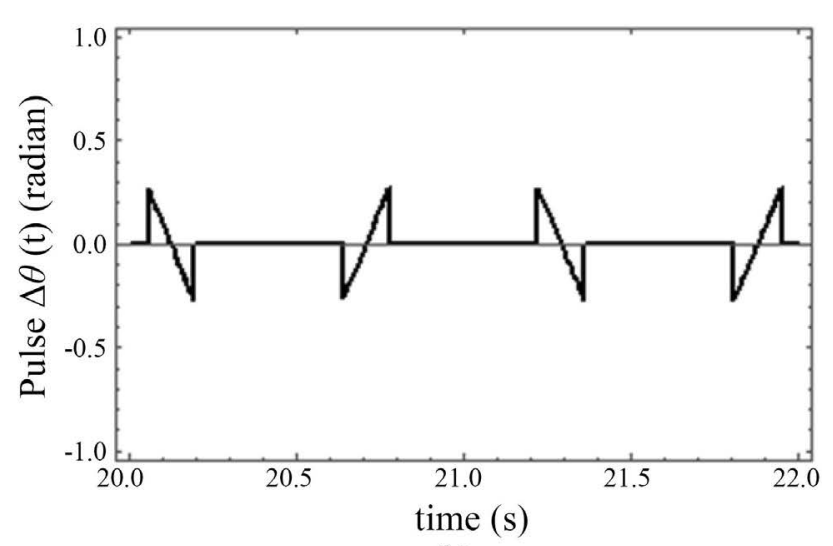

(b)

Figure 5. A magnified view of DB's motion and pulsation angles, $\Delta \theta(t)$, within $20.0 \leq t \leq 22.0$ seconds. (a) The angle $\theta(t)$ (rad) of the drinking bird's motion; (b) The pulsation angles extracted from the periodic motion of (a).

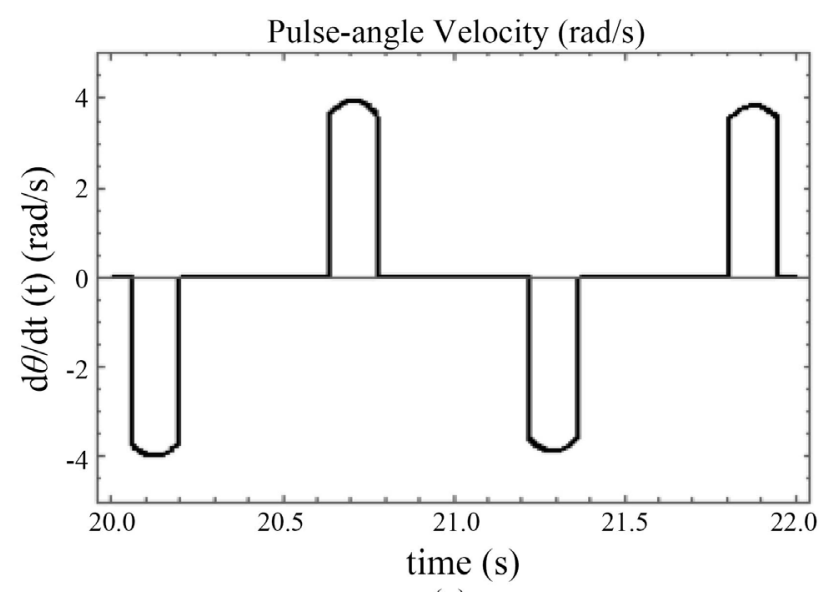

(a)

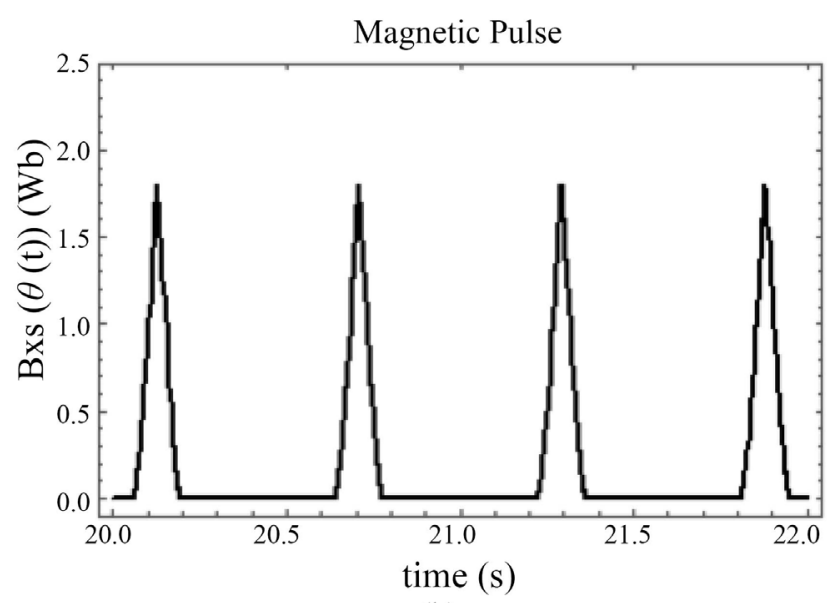

(b)

Figure 6. The magnetic pulse of DB's drinking motion and the angular velocity. (a) The alternating pulse angle-velocity; (b) The pulse of magnet flux: $\phi(t)=B S(\theta(t))$. 
The larger angular speed results in smaller response of magnetic flux. In other words, the coil could not "see" or take in the change of enough magnetic flux to produce the electric current when the angular speed, $\mathrm{d} \theta(t) / \mathrm{d} t$, becomes too large. There may exist a maximum and finite electric current generation in DM-EIM, which is checked in an experimental measurement. The optimal or maximum electric current and power is determined by physical parameters, such as the radius of magnet, strength of magnets, the number of windings of coil and resulting angular speed $\mathrm{d} \theta / \mathrm{d} t$, which depends on a mechanoelectric conversion system.

The pulse magnetic flux, $B S(\theta(t))(\mathrm{Wb})$, has a constant maximum, and the sharp magnetic-flux pulses produced by the DB's oscillations are shown in Figure $6(\mathrm{~b})$. Because the pulse angle velocity with $\left(\theta(0)=\pi / 3, \theta^{\prime}(0)=0\right)$ is larger than that of $\left(\theta(0)=\pi / 6, \theta^{\prime}(0)=0\right)$, the pulse magnetic flux with $\theta(0)=\pi / 3$ becomes a sharper spike than that of $\theta(0)=\pi / 6$, but the time range of the magnetic flux becomes smaller, resulting in a slow increase of electric currents and powers.

The pulses of electric current, $I(t)$, and electric power are shown in Figure 7. The pulses of electric currents change from -0.3 to 0.3 (ampere), according to the direction of DB's back and forth motion as shown in Figure 6. The pulses of electric currents with the starting value, $\theta(0)=\pi / 6$, change from -0.1 to 0.1 (ampere).

The pulses of the electric power change from -35 to 35 (watt) in Figure 7, which is usable for lighting LEDs (light emitting diodes). The pulses of electric power with the starting value, $\theta(0)=\pi / 6$, change from -4 to 4 (watt). The computer simulations with the disk-magnet electromagnetic induction with the assumption of the sharp magnetic-flux produce a moderate electric power characteristic to a system. Despite a limit of electromagnetic current generation, it would be useful for safe and sustainable energy generations and could be employed for engineering applications.

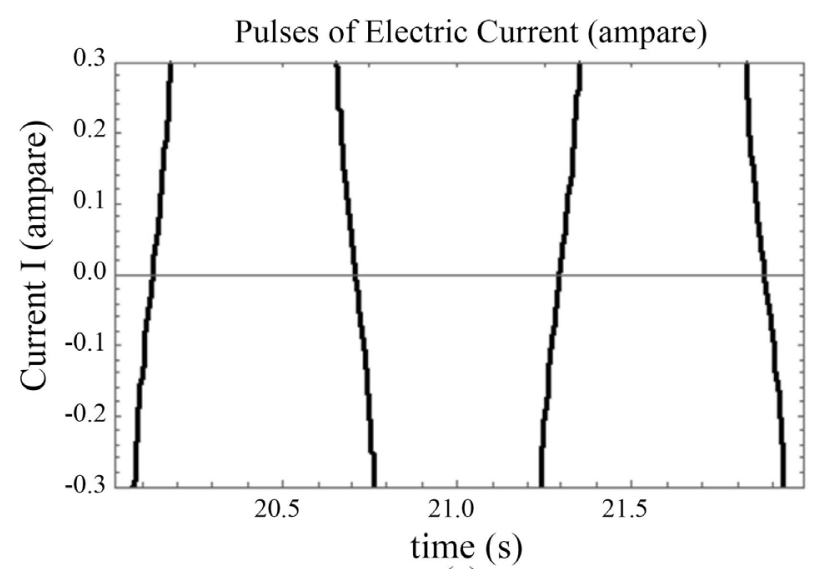

(a)

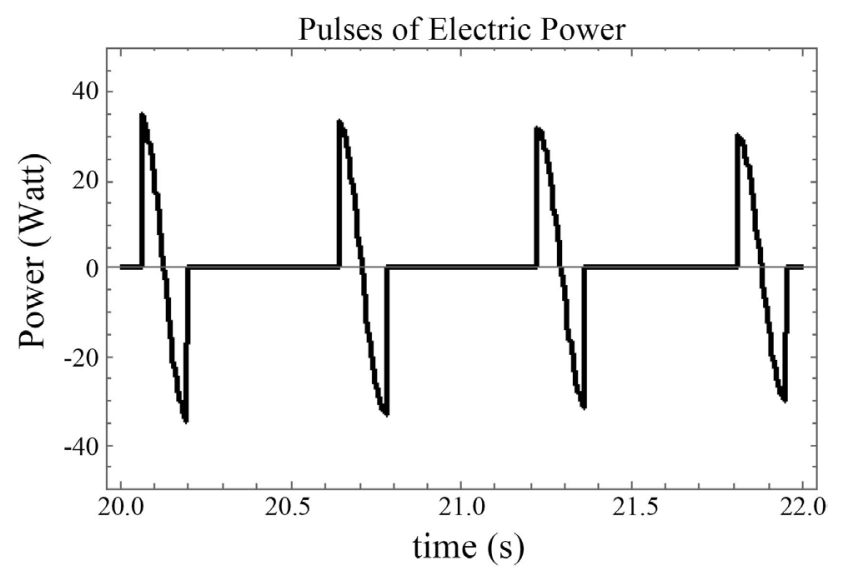

(b)

Figure 7. The produced pulsating current and power of DB's drinking motion. (a) The alternating pulse current; (b) The power generated by DB's motion. 


\subsection{The Disk-Magnet Electromagnetic Induction (DM-EMI)}

The results shown in the numerical simulations and DM-EMI technique should be considered qualitative and checked quantitatively by experiments and applied to direct mechanoelectric energy conversions, such as wind turbine systems, Stirling engines, even to coal-fired and nuclear radiation plants as one of energy harvesting technologies. Possible basic extensions of the disk-magnet electromagnetic induction (DM-EMI) could be applied to, for example, technique for heat-energy conversions of coal-fired and decommissioned nuclear plants. The sensitive energy-conversion technique derived from a water drinking bird would provide us with an important ideas and impacts to heat engines in our social life [10].

A generalization of DM-EMI is shown in Figure 8. The four disk magnets are positioned on a rotator, and the rotator moves among symmetrically positioned four coils (4DM-EMI). Each coil of right and left disks generates pulses of electric currents and powers. Similarly, one can introduce the 8 disk-magnet electromagnetic induction (8DM-EMI) and so on. When an angular speed increases or pulse responses in time become shorter and shorter, the direct numerical evaluations of DM-EMI may become difficult. One should note that an optimal angular speed would exist, resulting in maximum pulses of electric currents and powers, which should be experimentally checked with physical parameters of a specific mechanical system.

\section{Discussions and Perspectives}

Because the dynamics of a drinking bird and a Stirling engine is a very sensitive physical problem of the irreversible thermodynamics, it helps us understand natural phenomena in terms of energy and heat flows, resulting in conspicuous applicability and testability. The heat engine suggests that energy can be more efficiently produced and used so that the waste of energy can be dramatically
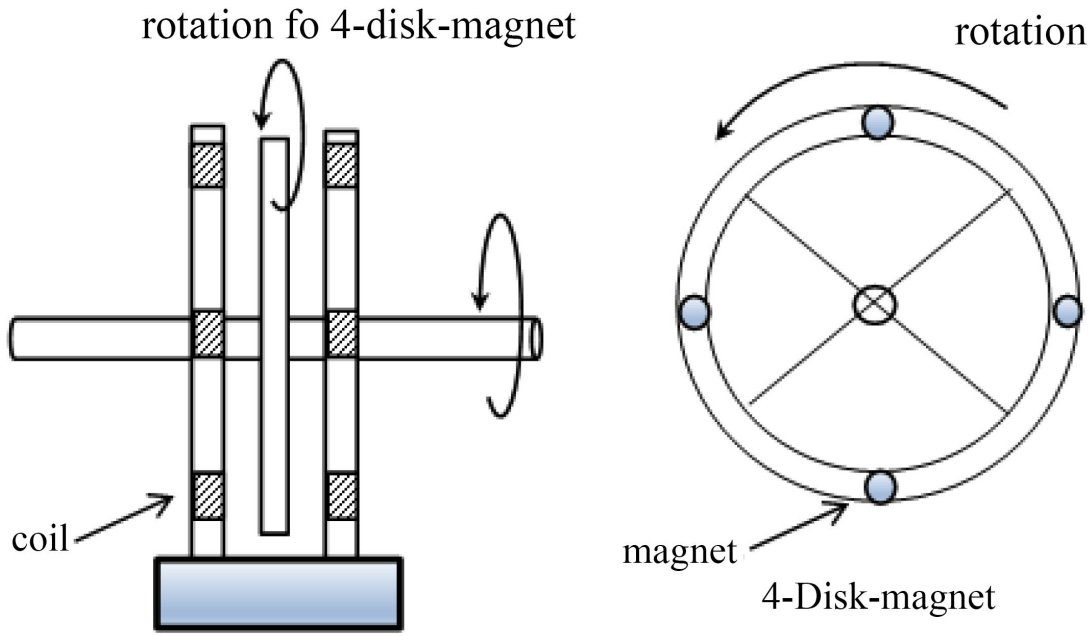

Figure 8. The application of 4-disk-magnet and 4-coil electromagnetic induction (4DMEMI). 
decreased. The structure of a drinking bird and a Stirling engine as heat engines could advance technologies for our energy productions and consumptions into more energy efficient systems.

Energy efficiencies of heat engines should be compared to those of plants and animals, such as photosynthesis of mitochondria, adenosine triphosphate (ATP), etc, as a source of heat and electric energy conversion system employed by biochemical processes. In views of science, drinking birds, Stirling engines, mitochondria and coal-fired plants, even nuclear power stations are regarded as having similar mechanism as heat engines using energy or conversions of heat flow to electric energy. Precisely speaking, systems mentioned above should be generally explained as transport engines, and even milli-watt to 100 -watt electric power can be extracted from warm water discharged from nuclear power plans to rivers and oceans at the end operations. Hence, the sensitive mechanism of plant and animals as transport engines would be very useful for the progress of modeling technologically efficient heat engines.

The sensitive heat engines have guided us to the idea of a direct mechanoelectric conversion technique, which should be applied mechanical rotation and heat transport systems in our daily life, such as horizontal and vertical wind turbines on housetops, vibration and rotation systems of automobiles [6] [7]. The generalization of disk-magnet electromagnetic induction, 4DM-EMI, 8DM-EMI, ..., rotating disk, would contribute to practical applications for energy harvesting technologies. The rectification and energy storage of pulse-power of EMI should be consulted with experts in those fields [11] for efficient use of DM-EMIs, and in addition, advanced thermal machines and technologies in the field of cryogenics [12], Stirling coolers [13], pulse-tube-refrigerators [14], thermoacoustic engines [15] and so forth, could be applied to make refined heat engines for sustainable and renewable energies.

We solved the equation of motion mathematically in the first paper on drinking bird's motion and explained fundamental logical structure of science, regarding reproducibility, self-consistency and testability [1]. The numerical experiment or reproducibility is exhibited in detail by nonlinear equation of motion with time-dependent coefficients [1]. The current paper is regarded as testability or applicability of sensitive irreversible thermodynamic mechanism of a drinking bird as transport engines, and we are directed to investigate self-consistency of our thermomechanical model of drinking birds in terms of entropy-flow in irreversible thermodynamics. It is to investigate physical reasons why a nonlinear model with time-dependent coefficients worked reasonably well, which will be pursued in following research.

The symmetric settings of right and left coils of DM-EMI are essential to avoid braking mechanism of EMI, and the optimal electric power production and energy efficiency of disk-magnet electromagnetic induction should be checked. The technology can be applied to environmental problems to support clean and sustainable energy (CSE) [10]. We will apply the direct mechanoelectric conversion method for resolving nuclear radiation wastes in the near future. 


\section{Acknowledgements}

We acknowledge Dr. Akihiko Nishimura of Japan Atomic Energy Agency (JAEA) for his kind and consistent support to our work.

\section{Conflicts of Interest}

The authors declare no conflicts of interest regarding the publication of this paper.

\section{References}

[1] Uechi, S.T., Uechi, H. and Nishimura, A. (2019) The Analysis of Thermomechanical Periodic Motions of a Drinking Bird. World Journal of Engineering and Technology, 7, 559-571. https://doi.org/10.4236/wjet.2019.74040

[2] Demirel, Y. (2014) Nonequilibrium Thermodynamics. Elsevier, Amsterdam. https://doi.org/10.1016/B978-0-444-59557-7.00003-5

[3] Førland, K.S., Førland, T. and Ratkje, S.K. (1988) Irreversible Thermodynamics; Theory and Applications. John Wiley \& Sons, Hoboken.

[4] Munoz, A.G. (2013) Photoelectrochemical Solar Conversion Systems, Molecular and Electronic Aspects. Taylor \& Francis Group, Abingdon-on-Thames.

[5] Compton, R.G. and Hancock, G. (1998) Chemical Kinetics. Elsevier Science, Amsterdam.

[6] Uechi, S.T. and Uechi, H. (2018) The Profiling of International Roughness Index (IRI) Based on Lagrangian Method. World Journal of Engineering and Technology, 6, 885-902. https://doi.org/10.4236/wjet.2018.64059

[7] Uechi, S.T. and Uechi, H. (2019) A Mechanical Vibration-Induced Electric Energy Generation (MVEG) and Applications to Ride Quality of Vehicles and International Roughness Index (IRI). Studies in Engineering and Technology, 6, 59-69.

https://doi.org/10.11114/set.v6i1.4301

[8] Henri Poincaré, Bifurcation Theory, (Wikipedia). https://en.wikipedia.org/wiki/Bifurcation,

[9] Lorenz, R. (2006) Finite-Time Thermodynamics of an Instrumented Drinking Bird Toy. American Journal of Physics, 74, 677. https://doi.org/10.1119/1.2190688

[10] Uechi, H. and Nishimura, A. (2020) Conversion of Nuclear Wastes into Clean and Sustainable Energy. LSSE 2020 Invited Talk, Photonics International Congress, Yokohama. https://lsse.opicon.jp/invited-talks

[11] Karady, G.G. and Holbert, K.E. (2013) Electrical Energy Conversion and Transport. John Wiley, Hoboken. https://doi.org/10.1002/9781118498057

[12] Radebaugh, R. (2009) Cryocoolers: The State of the Art and Recent Developments. Journal of Physics. Condensed Matter, 21, Article ID: 164219. https://doi.org/10.1088/0953-8984/21/16/164219

[13] Song, C., et al. (2015) Study on the COP of Free Piston Stirling Cooler (FPSC) in the Anti-Sublimation $\mathrm{CO}_{2}$ Capture Process. Renewable Energy, 74, 948. https://doi.org/10.1016/j.renene.2014.08.071

[14] Liang, J., et al. (1990) Development of a Single-Stage Pulse Tube Refrigerator Capable of Reaching 49 K. Cryogenics, 30, 49-51. https://doi.org/10.1016/0011-2275(90)90114-R

[15] Tijani, M.E.H. and Spoelstra, S. (2011) A High Performance Thermoacoustic Engine. Journal of Applied Physics, 110, Article ID: 093519. https://doi.org/10.1063/1.3658872 


\section{Appendix: The Energy Generation from Swinging Motion of a Drinking Bird by Employing the Disk-Magnet Electromagnetic Induction (DM-EMI)}

Let us calculate the time-dependent crossing surface, $S(\theta)$, which is shown as the shaded area in Figure 9. The length $R$ is given by $R(\theta(t))=a \sin (\theta / 2)$, and the height $h$ and $r$ are defined as shown in Figure 9. Since the height, $h$, is given by $h=\left(b^{2}-(R+r)^{2}\right)^{1 / 2}$, the shaded area of crossing, $S(\theta)$, is:

$$
S(\theta)=2 \int_{0}^{b-R} 2 h \mathrm{~d} r=4 \int_{R}^{b} \sqrt{b^{2}-x^{2}} \mathrm{~d} x
$$

where the change of variable, $x=r+R$, is used in the second expression, and a well known irrational integration formula gives

$$
\begin{aligned}
S(\theta) & =2\left[x \sqrt{b^{2}-x^{2}}+b^{2} \sin ^{-1}\left(\frac{x}{b}\right)\right]_{R}^{b} \\
& =b^{2} \pi-2 R \sqrt{b^{2}-R^{2}}-2 b^{2} \sin ^{-1}\left(\frac{R}{b}\right) .
\end{aligned}
$$

where $R(\theta)=a \sin (\theta / 2)$ and $R(\theta) \leq b$, and there is no crossing area when the length, $R(\theta)>b$ defined by $R(\theta) \equiv 0$ and $S(\theta) \equiv 0$. It is written in short as,

$$
R(\theta)=\left\{\begin{array}{lc}
a \sin (\theta / 2) & |\theta| \leq \sin ^{-1}(2 b / a) \\
0 & |\theta|>\sin ^{-1}(2 b / a) \text { and } S(\theta)=0
\end{array}\right.
$$

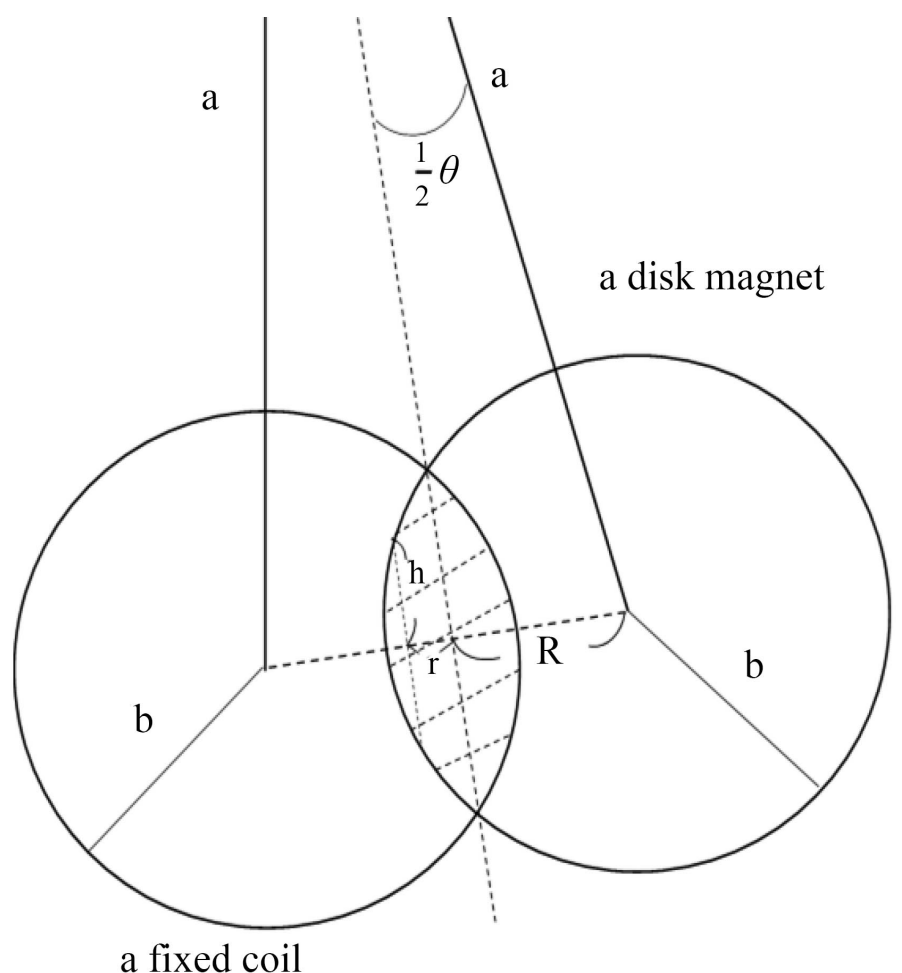

Figure 9. The image at a moment of an intersection for the coil cross-section and diskmagnet. The shaded area is $\phi(t)=B S(\theta(t)) \quad(\mathrm{Wb})$, and $R(t)=a \sin (\theta(t) / 2) \quad(\mathrm{cm})$. 
The change of area with respect to the angle $\theta, S(\theta) / \mathrm{d} \theta$, is calculated from Equation (A2), resulting in:

$$
\begin{aligned}
& \frac{\mathrm{d} S(\theta)}{\mathrm{d} \theta}=\left\{-2 a \sqrt{(b / a)^{2}-(R / a)^{2}}+\frac{2\left(R^{2} / a\right)}{\sqrt{(b / a)^{2}-(R / a)^{2}}}\right. \\
& \left.-\frac{2\left(b^{2} / a\right)}{\sqrt{(b / a)^{2}-(R / a)^{2}}}\right\}(\mathrm{d} R / \mathrm{d} \theta),
\end{aligned}
$$

where $\mathrm{d} R(\theta(t)) / \mathrm{d} \theta=(a / 2) \cos (\theta(t) / 2)$. Then, the magnetic flux, $\phi(\theta(t))=B S(\theta(t))$, is calculated, resulting in the induced potential, $V_{\phi}=-n \mathrm{~d} \phi / \mathrm{d} t \quad(n$ is the number of windings of a coil). The induced electric current is connected to a RLC circuit, for instance, as:

$$
V_{\phi}(\theta(t))+R \dot{Q}(t)+Q(t) / C=0,
$$

where $\dot{Q}(t), R$ and $C$ are the electric current, resistance and capacitance, respectively. The equation is solved simultaneously with (2.5) for $\theta(t)$ by setting initial conditions $\theta(0)$ and $\theta^{\prime}(0)$ (see the paper [1] to obtain solutions). In addition, the solution should be confined with (A3), and then, pulse angles, electric currents and powers are obtained. The electric currents and powers depend on physical parameters, $n, R, C$, the radius of magnet and coil, $b$, strength of magnet, $B$, and angular velocity, $\mathrm{d} \theta / \mathrm{d} t$. It is important to know that there exists a maximum electric currents and powers to be produced determined by energy levels of a mechanical system. The disk-magnet electromagnetic induction (DM-EMI) will produce a finite magnitude of electric currents and powers, which will be useful for applications in energy harvesting technologies. 